\title{
Oesophago-cardiac fistula complicating achalasia
}

\author{
R. W. STRONG \\ F.R.C.S.
}

Princess Alexandra Hospital, Brisbane, Australia, 4102

\begin{abstract}
Summary
A case is reported of a patient with carcinoma of the oesophagus, secondary to a longstanding achalasia and subsequent development of a fistulous communication between the oesophagus and the heart. The literature is reviewed.
\end{abstract}

\section{Introduction}

Fistulous communications between the heart and the oesophagus are extremely rare. They may be oesophago-pericardial, of which nineteen cases have been recorded, or oesophago-cardiac of which only two cases have been recorded. The communication in the oesophago-cardiac cases has been between the oesophagus and left atrium. In the case reported here, both communications occurred.

\section{Case report}

Miss A.C., aged 56 years, was referred to hospital by her doctor with general ill health and weight loss.

At the age of 26 years, the patient developed dysphagia. A barium meal was carried out, following which oesophagoscopy and dilatation were performed. She was then given a bougie which she used over the subsequent 6-12 months. From then until her present illness she was well. However, on closer questioning, she did admit to bouts of difficulty in swallowing, which seemed to clear spontaneously if she placed herself temporarily on a fluid diet.

Her present history began 6 weeks before admission, when she developed general malaise. Two weeks later, she developed a chest infection, for which she received an antibiotic and a linctus from her practitioner, and her cough improved. Soon after, she noticed a throbbing pain in the left loin, and this persisted until her admission. She began to regurgitate undigested food and mucus, 2 weeks before referral. There was no blood or bile in the vomit. There was associated constipation. A loss of 1-2 stone in weight had occurred over the 6 weeks.

On examination, she was an ill looking woman, who showed evidence of weight loss. She did not appear anaemic or dehydrated. Her pulse was $100 / \mathrm{min}$, with sinus rhythm; temperature $37.5^{\circ} \mathrm{C}$. The blood pressure was normal. The heart sounds were faint, but normal in character. The jugulovenous pressure was not raised. There was dullness to percussion and reduced breath sounds at the left base, together with generalized rhonchi. On examination, the abdomen and central nervous system were normal.

Chest X-ray (Fig. 1) showed a fluid level in the right paratracheal region. This was thought to be a dilated oesophagus, possibly as a result of achalasia. Her haemoglobin was $11.3 \mathrm{~g} \%$. White blood count $7500 / \mathrm{cc}$. ESR $10 \mathrm{~mm} / 1 \mathrm{hr}$. The electrolytes and urea were normal. A gastrografin swallow (Fig. 2) demonstrated a grossly dilated and tortuous oesophagus, with the lower end having a smooth tapering entry into the stomach.

There seemed no doubt that she had suffered from achalasia of the oesophagus for many years and would need surgery, but, because of the severe deterioration in her general condition, complicating carcinoma was suspected and a preliminary oesophagoscopy planned. As preparation for this, her oesophagus was aspirated and washed out with saline twice daily. She was restricted to clear fluids only by mouth, and her intravenous feeding included Aminosol and intralipid. She was given physiotherapy for her chest. The oesophageal residue decreased, and the return became clearer.

Four days after treatment was instituted, she was observed to have atrial fibrillation. There was no obvious predisposing cause. She was digitalized, and 2 days later returned to sinus rhythm. Forty-eight hours following her reverting to sinus rhythm, there was a dramatic change in the course of events. The patient became confused, and had a rigor. Her temperature rose to $38^{\circ} \mathrm{C}$. She was again in atrial fibrillation, and her systolic blood pressure fell 40 points to $100 \mathrm{~mm}$. Examination did not reveal any new abnormal signs. Chest X-ray, urine analysis and ultrasound testing of the legs were all normal. Blood cultures were negative.

Over the next 4 days, she remained unwell. Her 
Fig. 1. Chest X-ray, (a) A.P. and (b) lateral, showing fluid level in right paratracheal region.
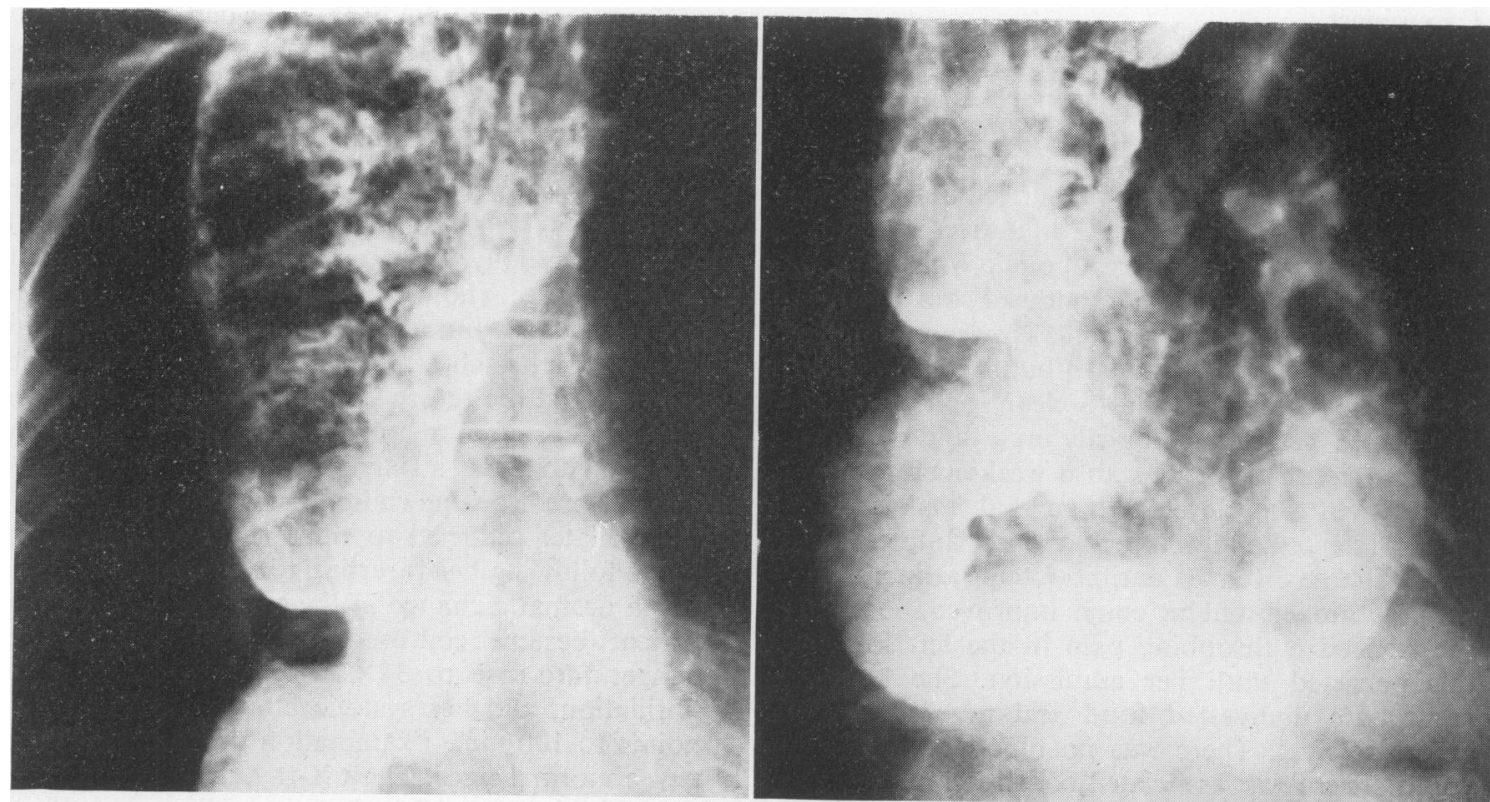

FIG. 2. Gastrografin swallow, showing (a) gross dilatation and tortuosity of oesophagus. The lower end (b) narrows at the cardia. 
temperature returned to normal, but her blood pressure continued to be low. At this stage her white count rose to $21,500 / \mathrm{cc}$ and the ESR to $56 \mathrm{~mm}$. She again developed a pyrexia, became confused, and the blood pressure fell even lower. She gradually became comatose, and at this stage auscultation revealed a pericardial rub. Her condition continued to deteriorate, and she died $12 \mathrm{hr}$ after lapsing into coma.

\section{Post mortem}

The oesophagus was grossly dilated, with a constriction about the level of the tracheal bifurcation. The lower end was narrow at the oesophago-gastric junction. There was a $6 \times 4 \mathrm{~cm}$ ulcer on the anterior wall of the oesophagus, the edges of the ulcer being adherent to the mediastinal tissues (Fig. 3). The floor of the ulcer opened into a mediastinal cavity which contained pus. There was a track leading from this cavity into the pericardial sac. The pericardial space contained $150 \mathrm{ml}$ of dark coloured fluid, with food particles evident. There was also a track leading on to the left atrium of the heart (Fig. 3b). The pericardium was thickened. There was a large left sided subphrenic abscess, in the retroperitoneal region, in relation to the left kidney and adrenal gland. The abscess was bounded above by the diaphragm, in which there was a $1.5 \mathrm{~cm}$ diameter opening into the left side of the chest, adjacent to the mediastinal structures. No direct communication could be found between the subphrenic and mediastinal abscesses. Posteriorly, the subphrenic abscess formed loculations in the quadratus lumborum and psoas major muscles. The brain showed abscesses in both frontal lobes.

Histological examination of the ulcer showed a moderate to poorly differentiated epidermoid carcinoma, infiltrating to a depth of $1 \mathrm{~cm}$. There were two perforations through the centre of the carcinoma -one into the pericardial cavity, and one into the left atrium. The adrenals showed involvement with carcinoma consistent with metastases from the oesophagus.

\section{Discussion}

The post mortem findings would seem to explain the clinical course of this patient. The gross dilatation and tortuosity of the oesophagus as seen on X-ray and at post mortem, were consistent with her 30year-history of difficulty in swallowing due to achalasia. Her final illness began with general malaise, which was soon followed by a cough and pyrexia, vomiting and left loin pain. Perforation of the carcinoma of the oesophagus, with formation of a mediastinal abscess, probably occurred at this stage, and its subsequent tracking down into the subphrenic retroperitoneal position on the left side, accounted for the left loin pain. However, direct communication between the two abscesses could not be demonstrated. The development of atrial fibrillation was evidence either of the development of pericarditis, or of the oesophago-atrial fistula. The cerebral abscesses undoubtedly arose as a result of this, and were heralded by the sudden collapse, rigor, hypotensive state and confusion.

The association of carcinoma of the oesophagus and achalasia is well established and widely reported (Just-Viera and Haight, 1969). There are, however, disparate figures regarding the incidence in the literature, varying from $1 \%$ to $20 \%$. Even accepting achalasia as a possible precursor to malignant change in the oesophagus, early diagnosis of a carcinoma is difficult. They are, therefore, frequently too advanced for surgical treatment. There have been many reports of tracheo-oesophageal fistulae terminally. Perforation of the oesophagus, resulting in a pneumothorax, empyema or pulmonary abscess may occur, and perforation of the aorta with massive bleeding can constitute the terminal stage.

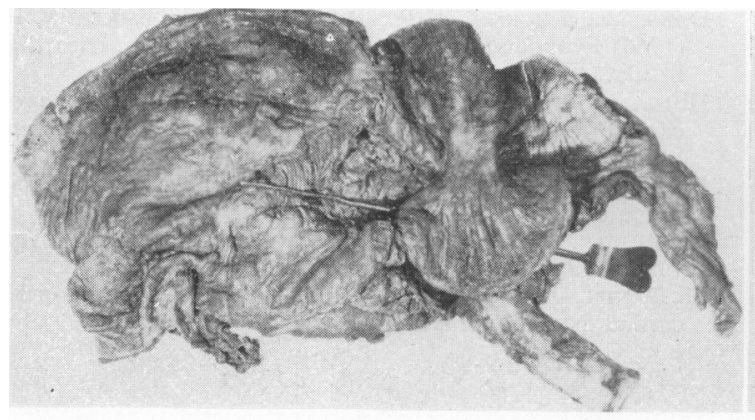

(a)

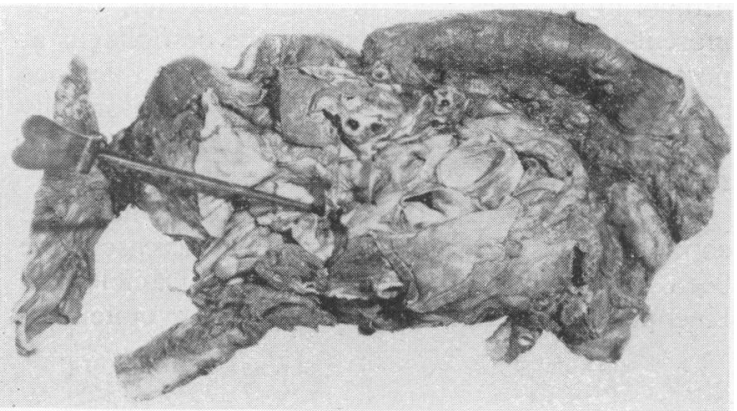

FIG. 3. (a) Post mortem specimen with large carcinoma of the oesophagus. Probe in position of oesophago-cardiac fistula. (b) Probe in orifice of fistula in left atrium. The orifice of the oesophago-pericardial fistula not demonstrated in this
photograph. 
In the patient reported here, perforation of the carcinoma resulted in the formation of a mediastinal abscess. This subsequently eroded into the pericardial cavity and left atrium, thus establishing the rare oesophago-cardiac and oesophago-pericardial fistulae. They have not previously been reported as a complication of achalasia.

Only two cases of oesophago-cardiac fistulae have been reported. In one (Laubscher, 1970), a 20-yearold man, there were no alimentary symptoms. He presented with a syncopal attack of short duration and no abnormal physical signs were elicited. He was found dead in his bed the next morning. Post mortem examination showed a chronic oesophageal ulceration, with a fistulous track leading to the left atrium. There was blood in the oesophagus. There was no evidence of hiatus hernia, reflux oesophagitis or peptic ulceration.

The other case (Hojgaard and Raaschou-Nielsen, 1970), a 73-year-old woman, had suffered from difficulty in swallowing for 12 years, and 18 months previous to her final illness, underwent surgery for hiatus hernia and stenosis. However she continued to experience difficulties in swallowing. The patient was admitted after a series of syncopal attacks. On examination she appeared to have suffered a myocardial infarct. She was anaemic. Her condition deteriorated, and the terminal phase was marked by a pyrexia, and non-characteristic cerebral symptoms. She died 6 days after admission. At post mortem she had a constricted area of oesophagus, adherent to mediastinal structures, with a small fistula leading from this area, into the left atrium. There were moderate amounts of blood in the oesophagus and stomach. She had fresh cortical infarcts of the occipital lobe of the brain.

The absence of overt bleeding seems surprising; although at post mortem examination, blood was found in the oesophagus in the two previously reported cases, there had been no haematemesis. The fistulae in all cases were of small diameter. In the present case the lack of blood in the oesophagus at post mortem was probably due to the difference between the left atrial pressure which is normally low, and the higher pressure in the oesophagus as a result of the column of retained fluid.

Oesophago-pericardial fistulae are somewhat more common, but still rare. Nineteen previous cases have been reported in the literature (McDaniel and Knepper, 1957; Dons et al., 1964). The pyopneumo- pericardium, like the oesophago-cardiac fistulae, $\frac{3}{2}$ carry a poor prognosis. One case, however, has been $\varnothing$ successfully repaired (Dons et al., 1964). There is a variable aetiology in the reported series. Five cases were due to carcinoma of the oesophagus. Five cases were caused by swallowing a foreign body, including a case of a swallowed denture and an occupational accident by a sword swallower. There have been five instances of benign idiopathic ulcers of the oesophagus, eroding into the pericardial sac. The remaining cases were of ulceration of a congenital tracheo-oesophageal diverticulum, and an attempted repair of a diverticulum of the lower oesophagus.

Provided early diagnosis can be established in oesophago-pericardial fistula, surgical repair is possible in benign cases. The prognosis in malignant cases is hopeless. Many of the cases have only been recognized at post mortem. The more frequent physical signs recorded are: precordial pain, splashing, churning, tinkling, metallic sounds, these murmurs synchronous with the heartbeat (McDaniel and Knepper, 1957). They may develop arrhythmias, as in this case. Pneumopericardium may be demonstrated on X-ray. Gastrografin swallow may demarcate the fistulous communication, although the track is usually very narrow, and therefore difficult to demonstrate.

The chances of diagnosing, and repairing a oesophago-cardiac are even more remote. The rariti of the condition, and the swiftness of its dire consequences, probably preclude successful diagnosis and treatment.

\section{Acknowledgments}

This patient was under the care of Mr A. York Mason and I am grateful for his permission to report this case, and for his helpful advice in the preparation of this paper. Also $\mathrm{Mr}$ T. W. Brandon, Department of Medical Photography, St Thomas' Hospital, for the photographic plates.

\section{References}

Dons, M., Ericksen, K.R., Ryssing, E. \& Therkelsin, F. (1964) Pyopneumopericardium with oesophagopericardial fistula. Acta Chirurgica Scandinovia, 128, 766.

HoJgAard, K. \& RAASChOU-Nielsen, T. (1970) Oesophagocardiac fistula. Acta Pathologica Mirobiologica Scandinavica, Suppl. 212, 114.

Just-VieRA, J.O. \& HAIGHT, C. (1969) Achalasia and carcinoma of the oesophagus. S.C.O. 1081.

LAUBSCHER, F.A. (1970) Oesophago-cardiac fistula. New England Journal of Medicine, 794.

MCDANiel, J.R. \& KNEPPER, P.A. (1957) Oesophago-pericardial fistula. Journal of Thoracic Surgery, 173. 